\title{
Reliability Analysis of Modified Irregular Augmented Shuffle Exchange Network (MIASEN)
}

\author{
Shobha Arya ${ }^{1}$ and Nipur Singh ${ }^{2}$ \\ 1,2 Department of Computer Science, Gurukul Kangri Vishwavidyalaya \\ Haridwar, Uttarakhand, India
}

\begin{abstract}
Multistage Interconnection Networks (MINs) play a vital role to accomplish high performance in the field of multiprocessing systems, parallel and distributed systems, networks-on-chips, broadband communications, and very large scale integration (VLSI) designs. A MIN is more reliable if it is able to handle the more faults encounter in different switching stages. In this paper, reliability of a MIN is investigated in terms of upper and lower bounds of Mean Time to Failure (MTTF) and a new network Modified Irregular Augmented Shuffle Exchange Network (MIASEN) has been proposed. The performance and comparison analysis shows that the proposed network is more reliable and fault tolerant than the existing Irregular Augmented Shuffle Exchange Network-2 (IASEN-2).

Keywords: Fault tolerance, reliability, multistage interconnection network.
\end{abstract}

\section{Introduction}

With advances in VLSI technology, a greater number of multiple-processor are used to accomplish high performance computation. There are two types of parallel computers interconnection networks: Static and Dynamic. The first one is a point-to-point connection network in which connections don't change during program execution while in case of later the connections are dynamically configured on demand of program. The dynamic interconnection networks are three types: Crossbar networks, bus networks, and Multistage Interconnection Networks. Multistage Interconnection Network (MIN) performs a vital role in high performance computing like supercomputers. MIN is used to create a connection among memory elements at one side and processing elements at other side connected by many stages of switching elements. The memory elements are used to store data required by the processing elements and processing elements are responsible for computational parallelism. MIN provides faster speed with low cost in a multiprocessor system as compared to single-processor system. These networks are used in both Single Instruction Multiple Data (SIMD) and Multiple Instruction Multiple Data (MIMD) computers. MINs can be two types: single path MINs and multi-path MINs. In single path MIN, there is one-to-one connection between each source and destination pair e.g. banyan network [1], baseline [8], butterfly [2], delta networks [5], binary n-cube network[6], omega network[7] and shuffle-exchange network. In a multi-path MIN there occurs one-to-many path connection between source and destination e.g. clos network [9], Parallel Benes [2], and Non-blocking extended generalized shuffle (EGS) network etc. Fault tolerance, and reliability are responsible for the performance of a MIN. single path MINs are less costly than multi-path MINs, but are less fault tolerant and reliable that is a major issue. Fault tolerance, reliability and permutation capability are the important issues and factors, which are able to measure the performance of a MIN.

A number of research works have been done to design new networks and to increase the fault-tolerance in MIN [3], $[4,5,6,7]$. Various routing schemes and permutation capability and other issues related to routing have also been broadly researched $[8,9,10]$, but a little research work has been done to the computation of reliability of these networks. Reliability is measured in terms of optimistic (or upper) bound and pessimistic (or lower) bound of Mean Time to Failure (MTTF). The simple series-parallel probabilistic combinations used to calculate reliability. In this research paper, a new MIN named Modified Irregular Augmented Shuffle Exchange Network (MIASEN) is has been proposed. The reliability of proposed Modified Irregular Augmented Shuffle Exchange Network (MIASEN) is compared with existing Irregular Augmented Shuffle Exchange Network-2 (IASEN-2).

The next section describes of design and basic structure of existing network and proposed Modified Irregular Augmented Shuffle Exchange Network (MIASEN). Section 3 focuses on the Fault-tolerance, and reliability aspects of MIASEN are analyzed. Section 4 concentrates on the cost, cost effectiveness of MINs is analyzed. In Section 5, the result and conclusion has been presented. 


\section{Structure and Design Of Multistage Interconnection Networks}

The dynamic MINs can be classified into three categories based on their topology: Regular, Irregular and Hybrid MINs.

In regular MIN, there equal number of switching elements (SEs) in each stage, but in irregular MIN, the number of switching elements (SEs) is not same in each stage. The hybrid MIN is the combination of regular and irregular MIN i.e. it consists the characteristics of both regular and irregular MIN. In this paper, we are focusing on irregular MINs. The existing Irregular Augmented Shuffle Exchange Network-2 (IASEN-2) and proposed Modified Irregular Augmented Shuffle Exchange Network (MIASEN) are discussed below.

\subsection{Irregular Augmented Shuffle Exchange Network-2 (IASEN-2)}

Irregular Augmented Shuffle Exchange Networks-2 (IASEN-2) [10] has $\mathrm{N}$ sources and $\mathrm{N}$ destinations with $\mathrm{n}=\left(\log _{2} \mathrm{~N}\right)$ stages. Each source and destination is associated with the multiplexers (MUX) of size $2 \times 1$ and demultiplexers (DEMUX) of size $1 \times 2$ respectively.

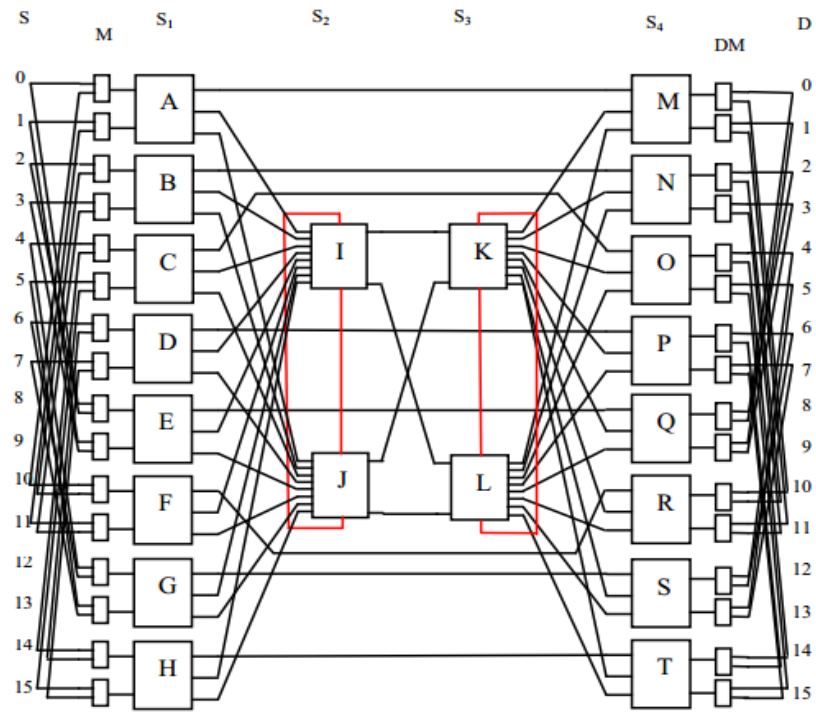

Fig. 1 Irregular Augmented Shuffle Exchange Networks-2 (IASEN-2).

The first and last stages are linked with N/2 switching elements (SE) [18]. The first stage and last stage have SEs of size $2 \times 3$ and $3 \times 2$ respectively but SE of second stage and third stage has size $9 \times 3$ and $3 \times 9$ respectively. The second and third stage consist N/8 SEs. The SEs of each stage is associated with each other through alternative links. The first and last stages are linked with N/2 switching elements (SE) [18].

\subsection{Modified Irregular Augmented Shuffle Exchange Network (MIASEN)}

The Modified Irregular Augmented Shuffle Exchange Network (MIASEN) is an $\mathrm{N} \times \mathrm{N}$ size irregular multistage interconnection networks with [( $\left.\left.\log _{2} \mathrm{~N}\right)-1\right]$ number of stages. First and last stages have N/2 switching elements (SE) and middle stage has (N/8) number of switching elements. MIASEN has $\mathrm{N}$ sources and $\mathrm{N}$ destinations, which are connected, with $\mathrm{N}$ multiplexers (MUX) and $\mathrm{N}$ demultiplexers (DEMUX) respectively. In the first stage, middle stage and last stage, size of each $\mathrm{SE}$ is $3 \times 3,5 \times 5$, and $2 \times 2$ respectively. The size of each multiplexer (MUX) and demultiplexer (DEMUX) in MIASEN is $2 \times 1$ and $1 \times 2$ respectively. In first stage, each switching element (SE) is attached with two multiplexer of size $2 \times 1$ and in last stage; two demultiplexers of size $1 \times 2$ are connected with each SE. The $16 \times 16$ network size MIASEN is mentioned in fig. 2.

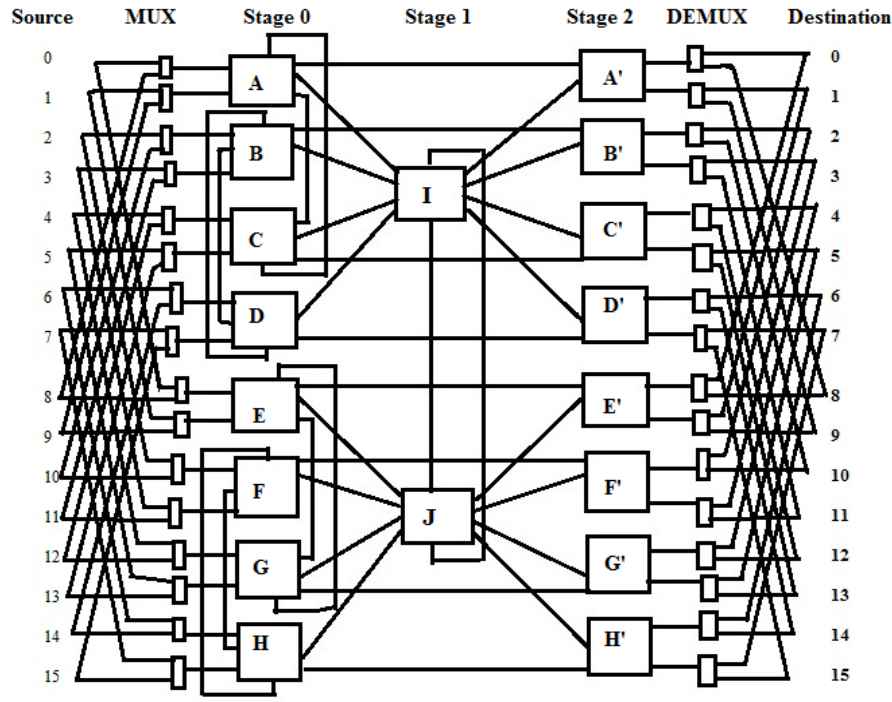

Fig. 2 Modified Irregular Augmented Shuffle Exchange Network (MIASEN).

A conjugate loop is formed when the switches are interconnected via the auxiliary links. The two switches, which form a loop, have their individual conjugate switches in an alternate loop for example, switches $A \& C$ and $\mathrm{B} \& \mathrm{D}$ are conjugate loops, and switches $\mathrm{A} \& \mathrm{~B}$ and $A \& D$ are conjugate switches in upper half of first stage of MIASEN [16]. The MIASEN is a fault tolerant and reliable MIN, if any failure occurs in any switch in the network then there will be an alternate path to work properly. MIASEN can be on-line repair and maintainable 
because if a loop is removed from any stage, MIASEN will work properly.

\section{Reliability Analysis of MIASEN and IASEN-2}

The reliability analysis can be basically considered as hardware reliability [12] and software reliability [11]. In this paper, the focus is given to the hardware reliability of the networks. There are three types of fault models implemented to measure reliability of MINs:

(1) stuck-at fault (2) link-fault (3) switch-fault

In Stuck-at fault, if any failure occurs in a crossbar switching element it'll remain in a particular state regardless of the control inputs given to it. This is affecting its capability to setup suitable connections.

In link-fault model, a failure affects a specific link of a switching element, leaving remaining part of the switch operational.

The switch fault model (or dead-fault model) is the worst case out of the three fault model. In this, if a switch fails then it'll become totally useless and non-operational [13].

These all fault model focus on the failure of switches and the failure effects on switches. The "full access" criterion and "switch fault" model is taken here to measure (MTTF) Mean Time To Failure of MIASENs. The full access means capacity to reach from any input to any output precisely in one pass even some switching components may be faulty (i.e. crossbar switches, MUX, DEMUX) but not the entire network [14] and this failure of components doesn't affects the reliability of others i.e. switch failure occurs independently. Reliability of IASEN-2, MIASEN networks is analyzed in terms of pessimistic (or lower) bound, optimistic (or upper) bound and Mean time to Failure (MTTF). Mean time to Failure (MTTF) is the estimated time elapsed before some source is separated from some destination [19]. Reliability equations of proposed MIASEN are derived in terms of MTTF lower bound and upper bounds and these bounds are calculated using simple series-parallel probabilistic combinations. To calculate reliability we need some assumptions for the analysis of the failure rates of the components, which are as follows:

- The failure rate of a segment can be derived from its gate count. For $2 \times 2$ crossbar switches, the failure rate is $\lambda$ (where $\lambda$ is about $10^{-6}$ per hour) [15].

- We assume that the failure rate of $m \times 1$ MUX and $1 \times \mathrm{m}$ DEMUX is $\mathrm{m} \lambda / 4$ i.e. $\lambda_{\mathrm{m}}=\lambda_{\mathrm{d}}=\mathrm{m} \lambda / 4$. Failure of the $2 \times 1$ MUX and $1 \times 2$ DEMUX occurs individually with failure rates of $\lambda_{\mathrm{m}}=\lambda / 2$ and $\lambda_{\mathrm{d}}=$ $\lambda / 2$ respectively.
- According to the adaptive routing scheme, the $2 \times 2$ switch in the last stage and its connected $1 \times 2$ DEMUX are considered in a series system. So we consider these three components as single segment $\left(\mathrm{SE}_{2 \mathrm{~d}}\right)$. Based on the gate count we assign failure rate to this group $\lambda_{2 \mathrm{~d}}=2 \lambda(2 \times 2=4$, $1 \times 2=2,1 \times 2=2$, total $=8,8 / 4=2$ ).

- Let failure rate of the $5 \times 5$ switch is $\lambda_{5}$ and $3 \times 3$ switch is $\lambda_{3}$, then based on gate count, $\lambda_{5}=6.25 \lambda$ and $\lambda_{3}=2.25 \lambda$ and $\lambda_{3 \mathrm{~m}}=3.25 \lambda$.

- Let failure rate of the $5 \times 5$ switch is $\lambda_{5}$ and $3 \times 3$ switch is $\lambda_{3}$, then based on gate count, $\lambda_{5}=6.25 \lambda$ and $\lambda_{3}=2.25 \lambda$.

\subsection{Optimistic or Upper Bound of MIASEN}

Each source is connected to two $2 \times 1$ MUX and each SE in the first stage has a conjugate pair in MIASEN. To calculate the upper bound we assumed that the MIASEN is working on condition that one of the two multiplexers attached to a source is operational and both components in a conjugate pair (switch or loop) are not faulty [14]. Therefore, we can say that even if the half of the component of a network (or one sub-network) is faulty even then MIASEN is still working. The upper bound block diagram of MIASEN is shown in fig. 3.

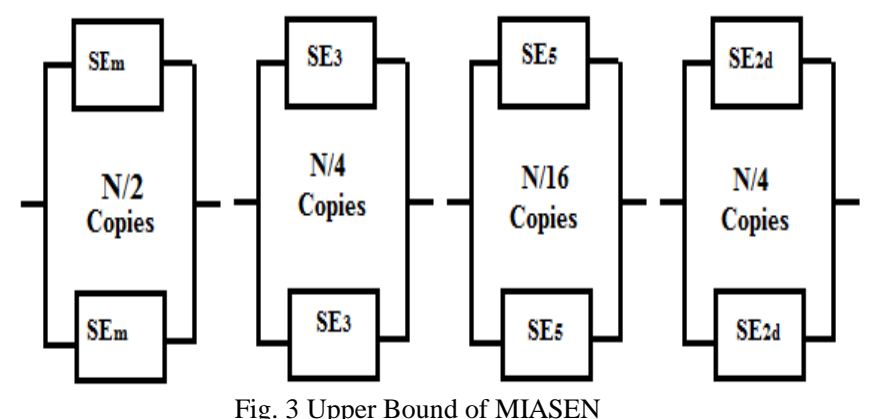

The reliability equations of upper bound are given below:

$$
\begin{aligned}
& \mathrm{f} 1=\left[1-\left(1-\mathrm{e}^{-\lambda_{\mathrm{m}} \mathrm{t}}\right)^{2}\right]^{\mathrm{N} / 2} \\
& \mathrm{f} 2=\left[1-\left(1-\left(\mathrm{e}^{-\lambda_{3} \mathrm{t}}\right)^{2}\right]^{N / 4}\right. \\
& \mathrm{f} 3=\left[1-\left(1-\mathrm{e}^{-\lambda_{5}} \mathrm{t}\right)^{2}\right]^{N / 16} \\
& \mathrm{f} 4=\left[1-\left(1-\mathrm{e}^{-\lambda_{2 \mathrm{~d}} \mathrm{t}}\right)^{2}\right]^{\mathrm{N} / 4}
\end{aligned}
$$

Where, $\lambda_{\mathrm{m}}=\lambda / 2, \lambda_{3}=2.25 \lambda, \lambda_{5}=6.25 \lambda, \lambda_{2 \mathrm{~d}}=2 \lambda$

$$
\mathrm{R}_{\text {MIASEN_UB }}=\mathrm{f} 1 * \mathrm{f} 2 * \mathrm{f} 3 * \mathrm{f} 4
$$

MTTF $_{\text {MIASEN-UB }}=\int_{0}^{00}$ RMIASENDUB(t) $\mathrm{dt}$ 


\subsection{Pessimistic or Lower Bound of MIASEN}

To calculate the pessimistic or lower bound of MIASEN, let us assume that the MIASEN is failed whenever more than one conjugate loop has a defective switch or multiple conjugate switch in the last stage fails. At the input side of MIASEN, routing algorithm doesn't consider the $2 \times 1$ MUX to be the integral part of the $3 \times 3$ switch. Therefore, if we group two MUX with each switch at input side and consider them a series system $\left(\mathrm{SE}_{3 \mathrm{~m}}\right)$ then we can say that if at least one of the MUX associated to a selected switch is working, the switch can still be used for routing [19]. The failure rate of $\mathrm{SE}_{3 \mathrm{~m}}$ is $\lambda_{3 \mathrm{~m}}=3.25 \lambda$. The block diagram of pessimistic or lower bound of MIASEN is shown in Fig. 4.

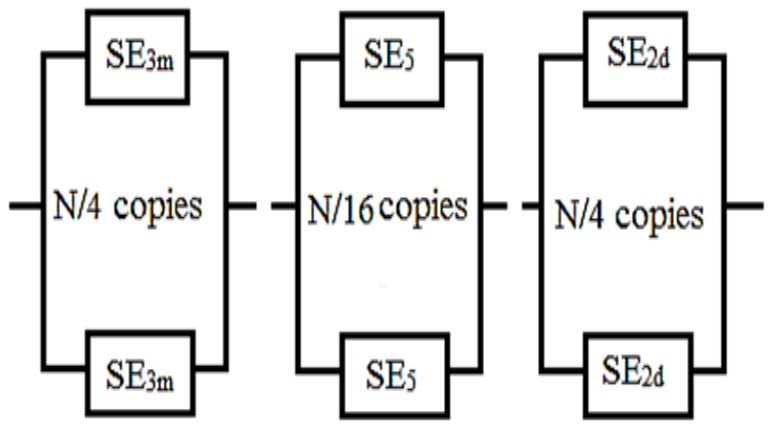

Fig. 4 Lower Bound of MIASEN

The reliability equations of lower bound are given below:

$$
\begin{aligned}
& \mathrm{f} 1=\left[1-\left(1-\mathrm{e}^{-\lambda_{3} \mathrm{~m}^{\mathrm{t}}}\right)^{2}\right]^{\mathrm{N} / 4} \\
& \mathrm{f} 2=\left[1-\left(1-\mathrm{e}^{-\lambda_{5} \mathrm{t}}\right)^{2}\right]^{\mathrm{N} / 16} \\
& \mathrm{f} 3=\left[1-\left(1-\mathrm{e}^{-\lambda_{2} \mathrm{dt}}\right)^{2}\right]^{\mathrm{N} / 4} \\
& \mathrm{R}_{\text {MIASEN_LB }}=\mathrm{f} 1 * \mathrm{f} 2 * \mathrm{f} 3
\end{aligned}
$$

Where, $\lambda_{3 \mathrm{~m}}=3.25 \lambda, \lambda_{5}=6.25 \lambda, \lambda_{2 \mathrm{~d}}=2 \lambda$

$$
\operatorname{MTTF}=\int_{0}^{\infty} \text { RMaAsen_Li }(\mathrm{t}) \mathrm{dt}
$$

\subsection{Optimistic or Upper Bound of IASEN-2}

Following the similar procedure as referred in the preceding segment (upper bound of MIASEN), the MTTF optimistic or upper bound of the IASEN-2 may be illustrated the use of the reliability block diagram shown in Fig. 5.

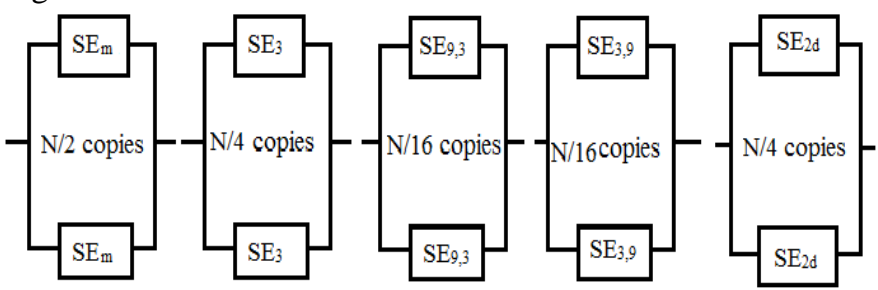

Fig. 5 Upper Bound of IASEN-2
Therefore, if we consider that the IASEN-2 is working so long as one of the two MUX connected to a switch is working and so long as a conjugate loop or conjugate switch isn't always faulty. Then we can certificates many as one half of the components to fail and the IASEN-2 might also still be working [14]. We can assume that the failure rate of $9 \times 3$ switching element $\left(\mathrm{SE}_{9,3}\right)$ is $\lambda_{9,3}$, failure rate of $3 \times 9$ switching element $\left(\mathrm{SE}_{3,9}\right)$ is $\lambda_{3,9}$ and failure rate of $2 \times 3$ switching element $\left(\mathrm{SE}_{2,3}\right)$ is $\lambda_{2,3}$. According to the gate counts of crossbar switch the failure rate of $\mathrm{SE}_{9,3}$ is $\lambda_{9,3}=6.75 \lambda$, the failure rate of $\mathrm{SE}_{3,9}$ is $\lambda_{3,9}=6.75 \lambda$ and failure rate of $\mathrm{SE}_{2,3}$ is $1.5 \lambda$.

Reliability equations for upper bound MTTF

$$
\begin{aligned}
& \mathrm{f} 1=\left[1-\left(1-\mathrm{e}^{-\lambda_{\mathrm{m}} \mathrm{t}}\right)^{2}\right]^{\mathrm{N} / 2} \\
& \mathrm{f} 2=\left[1-\left(1-\left(\mathrm{e}^{-\lambda_{2,3} \mathrm{t}^{2}}\right)^{2}\right]^{\mathrm{N} / 4}\right. \\
& \mathrm{f} 3=\left[1-\left(1-\mathrm{e}^{-\lambda_{9,3} \mathrm{t}}\right)^{2}\right]^{\mathrm{N} / 16} \\
& \mathrm{f} 4=\left[1-\left(1-\mathrm{e}^{-\lambda_{3,9} \mathrm{t}}\right)^{2}\right]^{\mathrm{N} / 16} \\
& \mathrm{f5}=\left[1-\left(1-\mathrm{e}^{-\lambda_{2} \mathrm{dt}}\right)^{2}\right]^{\mathrm{N} / 4}
\end{aligned}
$$

Where,

$$
\begin{gathered}
\lambda_{\mathrm{m}}=\lambda / 2, \lambda_{2,3}=1.5 \lambda, \lambda_{9,3}=6.75 \lambda, \lambda_{3,9}=6.75 \lambda, \lambda_{2 \mathrm{~d}}=2 \lambda \\
\mathrm{R}_{\mathrm{IASEN}-2 \_\mathrm{UB}}=\mathrm{f} 1 * \mathrm{f} 2 * \mathrm{f} 3 * \mathrm{f} 4 * \mathrm{f} 5 \\
\mathrm{MTTF}_{\text {IASEN-2_UB }}=\int_{0}^{\infty} \mathrm{R}_{\text {IASEN-2_UB }}(\mathrm{t}) \mathrm{dt}
\end{gathered}
$$

\subsection{Pessimistic or Lower Bound of IASEN-2}

Following the similar, procedure as we used before to calculate the MTTF lower bound of the MIASEN can be illustrated using the reliability block diagram shown in Fig.(6).

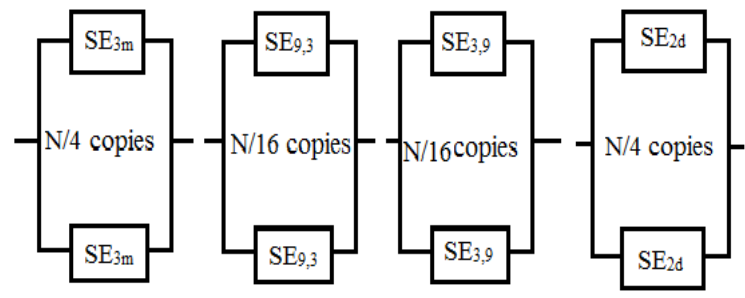

Fig. 6 Lower Bound of IASEN-2

Reliability equations for lower bound MTTF

Where,

$$
\begin{aligned}
& \mathrm{f} 1=\left[1-\left(1-\mathrm{e}^{-\lambda_{3 \mathrm{~m}} \mathrm{t}}\right)^{2}\right]^{\mathrm{N} / 4} \\
& \mathrm{f} 2=\left[1-\left(1-\mathrm{e}^{-\lambda_{9,3} \mathrm{t}}\right)^{2}\right]^{\mathrm{N} / 16} \\
& \mathrm{f} 3=\left[1-\left(1-\mathrm{e}^{-\lambda_{3,9} \mathrm{t}}\right)^{2}\right]^{\mathrm{N} / 16} \\
& \mathrm{f} 4=\left[1-\left(1-\mathrm{e}^{-\lambda_{22} \mathrm{t}}\right)^{2}\right]^{\mathrm{N} / 4}
\end{aligned}
$$

$$
\begin{aligned}
& \lambda_{3,9}=6.75 \lambda, \lambda_{9,3}=6.75 \lambda, \lambda_{2 \mathrm{~d}}=2 \lambda, \lambda_{3 \mathrm{~m}}=2.5 \lambda \\
& \mathrm{R}_{\mathrm{IASEN}-2 \_L B}=\mathrm{f} 1 * \mathrm{f} 2 * \mathrm{f} 3 * \mathrm{f} 4
\end{aligned}
$$

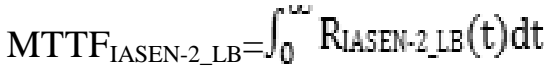


The Relative deviations in the MTTF upper and lower bounds of IASEN-2 and MIASEN, are illustrated in Fig. 7 and Fig. 8 respectively.

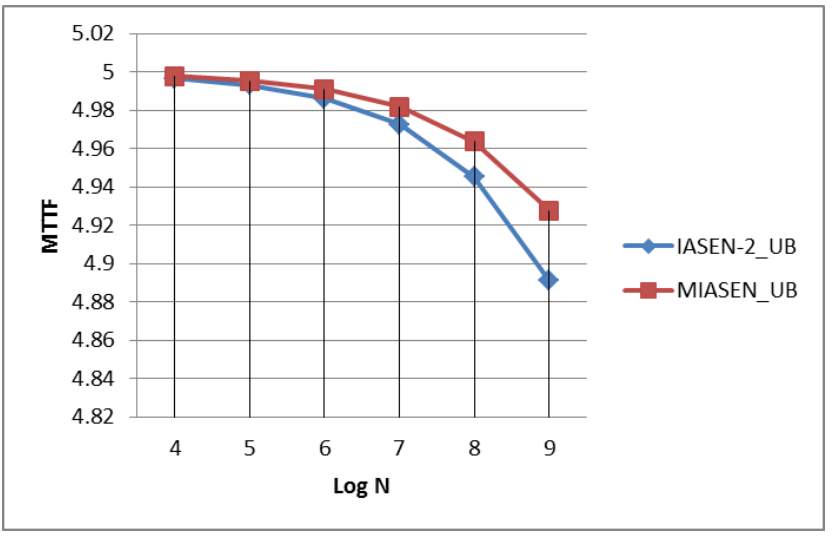

Fig. 7 MTTF Optimistic (Upper) bound comparison of MIASEN and IASEN-2

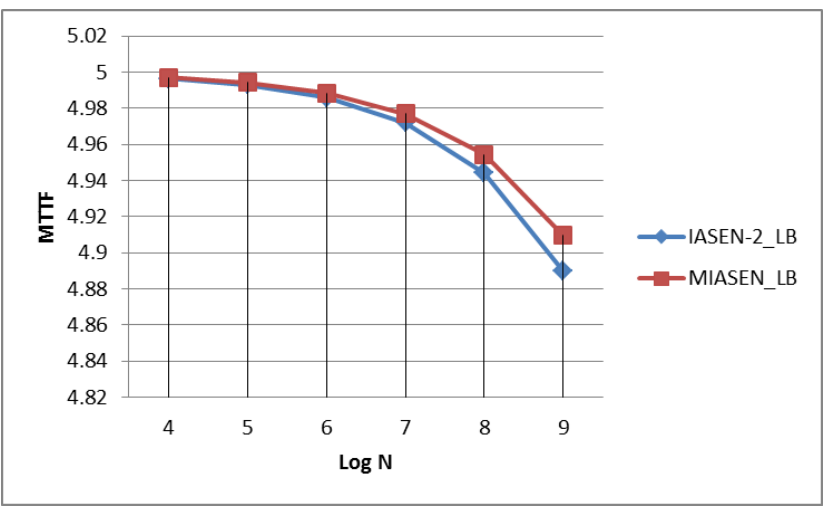

Fig. 8 MTTF Pessimistic (Lower) bound comparison of MIASEN and IASEN-2

The graph and table shows that the MTTF upper and lower bound of MIASEN are higher than the IASEN-2 for different network sizes $(\mathrm{N})$ (from $\mathrm{N}>=16$ and so on). The results of MTTF reliability equations are shown in table-1.

Table 1: MTTF Upper Bound and Lower Bound of IASEN-2 and MIASEN for different Network Size

\begin{tabular}{|c|c|c|c|c|}
\hline \multirow{2}{*}{$\begin{array}{c}\text { Network } \\
\text { Size } \\
(\operatorname{logN})\end{array}$} & \multicolumn{2}{|c|}{ IASEN-2 } & \multicolumn{2}{c|}{ MIASEN } \\
\cline { 2 - 5 } & $\begin{array}{c}\text { Upper } \\
\text { Bound }\end{array}$ & $\begin{array}{c}\text { Lower } \\
\text { Bound }\end{array}$ & $\begin{array}{c}\text { Upper } \\
\text { Bound }\end{array}$ & $\begin{array}{c}\text { Lower } \\
\text { Bound }\end{array}$ \\
\hline 4 & 4.9966 & 4.9964 & 4.9977 & 4.9971 \\
\hline 5 & 4.9931 & 4.9931 & 4.9954 & 4.9942 \\
\hline 6 & 4.986 & 4.9859 & 4.9909 & 4.9885 \\
\hline 7 & 4.9724 & 4.9719 & 4.9817 & 4.977 \\
\hline 8 & 4.945 & 4.9443 & 4.9637 & 4.9544 \\
\hline 9 & 4.8911 & 4.8898 & 4.9277 & 4.9095 \\
\hline
\end{tabular}

\section{Cost Analysis}

The cost a network can be calculated that the cost of a switch is number of gates involved which is proportional to the number of crosspoints within that switch [17]. For example, the cost of a $2 \times 2$ switch is 4 units of hardware. For mx1 MUX and 1xm DEMUX let the cost is $m$ unit of hardware. The cost of proposed MIASEN with network size $16 \times 16$ is given in the Table 2 .

Table 2: Cost of MIASEN

\begin{tabular}{|c|c|c|c|}
\hline $\begin{array}{c}\text { Type of } \\
\text { Component }\end{array}$ & Size & $\begin{array}{c}\text { Total No. of Switch } \\
\text { /MUX or DEMUX }\end{array}$ & Cost \\
\hline switch & $3 \times 3$ & 8 & $3 \times 3 \times 8=72$ \\
\hline Switch & $5 \times 5$ & 2 & $5 \times 5 \times 2=50$ \\
\hline Switch & $2 \times 2$ & 8 & $2 \times 2 \times 8=32$ \\
\hline MUX & $1 \times 2$ & 16 & $1 \times 2 \times 16=32$ \\
\hline DEMUX & $2 \times 1$ & 16 & $2 \times 1 \times 16=32$ \\
\hline \multicolumn{4}{|c}{ Cost of Overall MIASEN } \\
\hline
\end{tabular}

The cost of existing IASEN-2 with network size $16 \times 16$ is given in Table 3.

Table 3: Cost of IASEN-2

\begin{tabular}{|c|c|c|c|}
\hline $\begin{array}{c}\text { Type of } \\
\text { Component }\end{array}$ & Size & $\begin{array}{c}\text { Total No. of Switch } \\
\text { /MUX or DEMUX }\end{array}$ & Cost \\
\hline switch & $2 \times 3$ & 8 & $2 \times 3 \times 8=48$ \\
\hline switch & $9 \times 3$ & 2 & $9 \times 3 \times 2=54$ \\
\hline switch & $3 \times 9$ & 2 & $3 \times 9 \times 2=54$ \\
\hline switch & $3 \times 2$ & 8 & $3 \times 2 \times 8=48$ \\
\hline MUX & $1 \times 2$ & 16 & $1 \times 2 \times 16=32$ \\
\hline DEMUX & $2 \times 1$ & 16 & $2 \times 1 \times 16=32$ \\
\hline \multicolumn{4}{r}{ Cost of Overall IASEN-2 } \\
\hline \multicolumn{4}{r}{} \\
\hline
\end{tabular}

Thus, the cost of IASEN-2 is more than the cost of MIASEN.

\section{Conclusion}

In this paper, the proposed Modified Irregular Augmented Shuffle exchange Network (MIASEN) has accomplished significant fault tolerance and good reliability with relatively low cost as compared to IASEN-2. The results and analysis shows that MTTF upper and lower bound of MIASEN is always higher than that of IASEN-2. Therefore, we can say that MIASEN is having better reliability and more fault-tolerant than IASEN-2 network.

\section{Acknowledgments}

The authors would really like to thank the editor and the anonymous reviewers for his or her extremely good comments and reviews. Independently I, Shobha, would like to thank my parents, sisters, and husband who have been a pillar of energy for me and without whose encouragements none of this will were viable. In addition to this, I would like to thanks Professor Nipur Singh, 
whose knowledge, intelligence, and guidance continually a type of blessing for me.

\section{References}

[1] X. Jiang, A. Pattavina, and S. Horiguchi, "Strictly nonblocking f-cast photonic networks", IEEE/ACM Trans. Netw, Vol. 16, no. 3, pp. 73245, 2008.

[2] F. Bistouni, and M. Jahanshahi, "Improving the reliability of the Benes network for use in large-scale systems", Microelectron. Reliabil., Vol. 55, no. 3, pp. 67995, 2015.

[3] R. Aggarwal, and L. Kaur, "An efficient Routing Scheme to provide more Fault-tolerance for an Irregular Multistage Interconnection Network", Advance Computing Conference, IEEE International, 2009, pp. 948.

[4] F. Bistouni, and M. Jahanshahi, "Improved extra group network: a new fault-tolerant multistage interconnection network", J. Supercomput., Vol. 69, no. 1, pp. 16199, 2014.

[5] R. He, and J. G. Delgado-Frias, "Fault tolerant interleaved switching fabrics for scalable high-performance routers", Parallel Distribut. Syst. IEEE Trans., Vol. 18, no. 12, pp. 172739, 2007.

[6] C. C. Fan, and J. Bruck, "Tolerating multiple faults in multistage interconnection networks with minimal extra stages”, Comput. IEEE Trans., Vol. 49, no. 9, pp. 9981004, 2000.

[7] X. Shen, F. Yang, and Y. Pan, "Equivalent permutation capabilities between time-division optical omega networks and non-optical extra-stage omega networks", IEEE/ACM Trans. Netw., Vol. 9, no. 4, pp. 51824, 2001

[8] Y. Yang, and J. Wang, "Routing permutations on baseline networks with node-disjoint paths", Parallel Distribut. Syst. IEEE Trans., Vol. 16, no. 8, pp. 73746, 2005.

[9] P. H. Pham, J.Song, J.Park, and C.Kim, "Design and implementation of an on-chip permutation network for multiprocessor system-on-chip", Very Large Scale Integration (VLSI) Syst. IEEE Trans., Vol. 21, no. 1, pp. 1737, 2013.

[10] Ved Prakash Bhardwaj and Nitin, "On the Performance Analysis of IASEN-3 in Faulty and Non-faulty Networks Conditions", Proceedings of AASRI Conference on Intelligent Systems and Control, pp. 104-109, 2013.

[11] A. Sheta, "Parameter Estimation of Software Reliability Growth Models by Particle Swarm Optimization", ICGSTAIML Journal, Vol. 7, No.7, June,2007, pp.55-61.

[12] R. Aggarwal and L. Kaur, "On Reliability Analysis of Faulttolerant Multistage Interconnection Networks", International Journal of Computer Science and Security, Vol. 2, No. 4, August 2008, pp. 1-8.

[13] J. Nathaniel Davis, William, Tsun-yuk Hsu and H. J. Siegel, "Fault location techniques for distributed control interconnection networks", IEEE Transactions on Computers, Vol. C-34, No.10, October 1985.

[14] V. P. Kumar and S. M. Reddy, "Design and analysis of fault-tolerant multistage interconnection networks with low link complexity", Proceedings of 12th International Symposium on Computer Architecture, 1985 June, pp. 376386.

[15] Bansal, P. K., Joshi, R.C., Singh, K., "On a Fault tolerant Multistage Interconnection Network", International Journal of Electronics and Electrical Engineering, 20(4), pp. 335-345,1994.

[16] Aggarwal R., Aggarwal H. and Kaur L., "On Bandwidth analysis of Irregular Fault-tolerant Multistage Interconnection networks", International Review on Computers and Software, Vol. 3, No. 2, March 2008, pp. 199-202.

[17] Bansal P.K, Singh Kuldeep and Joshi R.C., "Quad Tree: A Cost-Effective Fault-Tolerant Multistage Interconnection network", Proceeding of International Conference IEEE INFOCOM, 1992, pp. 6D.1.1-6D.1.7.

[18] Ved Prakash Bhardwaj and Nitin, "A New Fault Tolerant Routing Algorithm for IASEN-2", Proceeding of second International Conference on Advances in Computing and Communications, pp199-202, 2012.

[19] Karamjit Kaur Cheema, Rinkle Aggarwal, "Design Scheme and Performance Evaluation of a new Fault-tolerant Multistage Interconnection Network", IJCSNS International Journal of Computer Science and Network Security, VOL.9 No.9, pp. 270-276, September 2009.

Er. Shobha Arya, received her Bachelor of Engineering degree in Computer Science \& Engineering from Kumaun Engineering College, Dwarahat, in 2006 and Master of Technology in Computer Science and Engineering from G. B. Pant Engineering College, Paudi, in 2012. Presently she is pursuing her Ph.D. in Computer Science from Gurukul Kangri Vishwavidyalaya, Haridwar. Her research interests include Interconnection Networks, Distributed Computing, and Parallel Processing.

Dr. Nipur Singh, Master of Computer Applications, PhD (Computer Science), is currently working as Professor in Gurukul Kangri Vishwavidyalaya, Haridwar. She has more than 15 years of teaching experience. She has supervised $6 \mathrm{PhD}$ Dissertations and contributed 39 articles in Conferences, 26 papers in research Journals and two chapters in book. Her areas of interest are Distributed Computing, Interconnection Networks, Mobile Agent Technology, Cloud Computing, Adhoc Networks. 